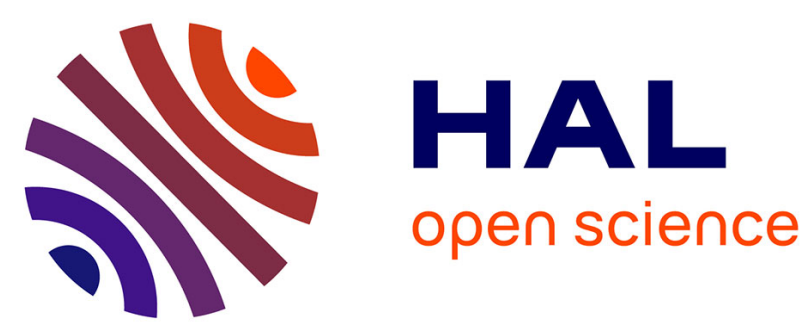

\title{
Etude de la sécrétion phéromonale de la tordeuse européenne de l'œillet: Cacoecimorpha pronubana (Hb.), Lep. Tortricidae, Tortricinae
}

Charles Descoins, Jacques Einhorn, Bernard Lalanne-Cassou, Madeleine Pralavorio, Nicole Deschamps, Martine Gallois

\section{To cite this version:}

Charles Descoins, Jacques Einhorn, Bernard Lalanne-Cassou, Madeleine Pralavorio, Nicole Deschamps, et al.. Etude de la sécrétion phéromonale de la tordeuse européenne de l'œillet: Cacoecimorpha pronubana (Hb.), Lep. Tortricidae, Tortricinae. Agronomie, 1985, 5 (1), pp.19-26. hal-00884728

\section{HAL Id: hal-00884728 https://hal.science/hal-00884728}

Submitted on 1 Jan 1985

HAL is a multi-disciplinary open access archive for the deposit and dissemination of scientific research documents, whether they are published or not. The documents may come from teaching and research institutions in France or abroad, or from public or private research centers.
L'archive ouverte pluridisciplinaire HAL, est destinée au dépôt et à la diffusion de documents scientifiques de niveau recherche, publiés ou non, émanant des établissements d'enseignement et de recherche français ou étrangers, des laboratoires publics ou privés. 


\title{
Etude de la sécrétion phéromonale de la tor- deuse européenne de l'œillet : Cacoecimorpha pronubana (Hb.), Lep. Tortricidae, Tortricinae
}

\author{
Charles DESCOINS, Jacques EINHORN, Bernard LALANNE-CASSOU \& Madeleine PRALAVORIO (*) \\ avec la collaboration technique de Nicole DESCHAMPS $\left({ }^{*}\right) \&$ Martine GALLOIS \\ I.N.R.A./C.N.R.S., Laboratoire des Médiateurs chimiques, Domaine de Brouessy, Magny-les-Hameaux, \\ F 78470 Saint-Rémy-lès-Chevreuse \\ (*) I.N.R.A. Station de Recherches de Zoologie et de Lutte biologique, 37, boulevard du Cap, F 06602 Antibes
}

RÉSUMÉ

Les constituants élémentaires présents dans la sécrétion phéromonale émise par les femelles vierges de Cacoecimorpha pronubana ont été identifiés par chromatographie en phase gazeuse, spectrométrie de masse et microchimie. Il s'agit de 6 composés dont 3 seulement jouent clairement un rôle dans le comportement sexuel du mâle : les acétoxy-1 tétradécène-11Z et 11E (Z-11 et E-11 TDA) et le tétradécène-11Z ol-1 (Z-11 TDol). Des capsules chargées de $1000 \mu \mathrm{g}$ d'un mélange : Z-11/E-11 TDA (97/3) : 90 p. $100 ; Z-11$ TDol : 10 p. 100 permettent de suivre l'évolution du vol de cette tordeuse dans les cultures florales du Sud-Est de la France. Les femelles de $C$. pronubana utilisent donc, comme celles des autres Archipini étudiés, le Z-11 TDA comme constituant majoritaire de leur sécrétion phéromonale.

Mots clés additionnels : Phéromone sexuelle, identification, piégeage. $(H b$.$) , Lep. Tortricidae, Tortricinae.$

Elementary components present in the sex pheromone blend produced by Cacoecimorpha pronubana virgin females were identified by gas-chromatography, mass spectrometry and microchemistry. There were 6 components but only 3 were definitely active on male sexual behavior : Z-11 and E-11 tetradecenyl acetate (Z-11 and E-11 TDA) and Z-11 tetradecen-1-ol (Z-11 TDol). Caps baited with a $1000 \mu \mathrm{g}$ blend of Z-11/E-11 TDA (97/3) : $90 \% ; Z-11$ TDol : $10 \%$ were used to monitor flights of this tortricid in field crops of flowers in Southeastern France. C. pronubana females, like all the other Tortricidae studied in the Archipini tribe, were found to use Z-11 TDA as the main component of its sex pheromone blend.

Additional key words : Sex pheromone, identification, trapping.

\section{INTRODUCTION}

La tordeuse européenne de l'œillet, Cacoecimorpha pronubana $(\mathrm{Hb}$.$) est une tordeuse Tortricinae de la$ tribu des Archipini. On la trouve maintenant non seulement dans toute l'Europe méridionale, son foyer d'origine (MILliÈRE, 1864), mais aussi dans la majeure partie de la France, le sud de l'Angleterre et l'Afrique du Nord où elle cause des dégâts aux citrus (DELUCCHI \& MERLE, 1962, cité par BOVEY, 1966).

Très polyphage, cette espèce peut se développer sur de nombreux végétaux spontanés ou cultivés mais elle s'est principalement installée sur les cultures d'œillets et de rosiers où elle fait des dégâts très importants et rend les fleurs coupées impropres à l'exportation. La chenille de $C$. pronubana ronge les feuilles des bourgeons principaux ou axillaires et les rassemble à l'aide de fils de soie provoquant des déformations caractéristiques en " crosse" ou en "bec d'oiseau ». Elle peut également pénétrer dans le bouton floral qui, rongé à l'intérieur, arrête son développement et se dessèche (TARGE \& DEPORTES, 1961).

La difficulté de la lutte contre cette tordeuse vient du fait que seul le stade jeune chenille est sensible aux traitements phytosanitaires non systémiques, les seuls utilisés dans la pratique. Aux stades plus âgés, la larve 
est en effet protégée à l'intérieur du végétal et l'intervention, à l'apparition des dégâts, ne peut alors plus donner aucun résultat satisfaisant.

Par ailleurs, plusieurs facteurs viennent compliquer la détermination de la date du traitement :

- La polyphagie de l'espèce qui permet sa conservation dans la nature et qui rend difficile la surveillance des populations ;

- Le polyvolţinisme avec chevauchement des générations ;

- La diversité des aires de cultures et des biotopes, entraînant des décalages à la sortie de l'hivernation, le $1^{\text {er }}$ vol pouvant ainsi s'étaler sur plusieurs mois.

Pour ces différentes raisons et afin d'améliorer la lutte contre ce ravageur, il était intéressant de disposer d'un moyen d'avertissement agricole sélectif et facile d'emploi, basé sur le piégeage sexuel des mâles par une phéromone de synthèse.

De plus, l'identification de cette phéromone permettrait de la comparer à celle des autres représentants de la même tribu et de mieux comprendre les bases chimiques de l'isolement entre espèces sympatriques.

\section{MATÉRIEL ET MÉTHODES}

Les insectes utilisés dans cette étude proviennent d'un élevage réalisé sur le même milieu semi-artificiel que celui mis au point pour Epichoristodes acerbella (Walk.) par PRALAVORIO \& MILLOT (1978), à partir de chenilles prélevées sur cultures d'œillets de la région d'Antibes. L'élevage est conduit en chambre climatisée sous photopériode de jours longs $(16 \mathrm{~h}$ de jour $/ 8 \mathrm{~h}$ de nuit), à $22 \pm 2{ }^{\circ} \mathrm{C}$ et $75 \pm 10$ p. 100 H.R.

Dans ces conditions, la durée totale du développement est de $40 \mathrm{j}$ et les générations se succèdent sans diapause en gardant une bonne vigueur.

Les sexes sont séparés à l'état de chrysalides et les imagos mâles et femelles, alimentés en eau sucrée à 10 p. 100 , sont conservés dans des enceintes séparées afin d'éviter tout contact entre eux.

La glande à phéromone, située sur la partie dorsale de la membrane intersegmentaire reliant les segments 8 et 9 , est prélevée sur des femelles vierges, âgées de 2 j, en période d'appel. Celles-ci prennent la posture d'appel (ailes légèrement relevées, derniers segments abdominaux dévaginés) $5 \mathrm{~h}$ environ après le début de la photophase et pendant $2 \mathrm{~h}$ environ.

Le prélèvement se fait à la manière habituelle en appuyant légèrement sur l'abdomen de la femelle pour en dévaginer les derniers segments et en coupant, sous loupe binoculaire avec des ciseaux d'ophtalmologie, l'extrémité abdominale de l'insecte au-dessus du $8^{\mathrm{e}}$ segment. On enlève ensuite les papilles anales et les fragments de corps gras pouvant encore adhérer à la préparation.

Le contenu volatil est analysé soit directement par chromatographie en phase gazeuse sans traitement préalable (DESCOINS \& GALLOIS, 1979), soit au contraire après extraction à l'hexane.

Les lavages de glandes ("wash » des auteurs anglosaxons) ont été réalisés en maintenant extrudée, par légère pression, la glande productrice de phéromone d'une femelle vierge en appel et en la plongeant pendant $10 \mathrm{~s}$ dans $10 \mu \mathrm{l}$ d'hexane. Cet extrait est ensuite immédiatement analysé par chromatographie en phase gazeuse (KLUN et al., 1979). La collecte des effluves phéromonaux produits par les femelles vierges en appel a été réalisée de la façon suivante : 100 femelles vierges sont introduites dans une enceinte cylindrique en verre ( $230 \mathrm{~mm}$ de long ; $120 \mathrm{~mm}$ de diamètre). A l'une des bases de ce cylindre est fixée une cartouche contenant $500 \mathrm{mg}$ de Porapak Q préalablement conditionné par chauffage sous azote pendant $24 \mathrm{~h}$ à $220^{\circ} \mathrm{C}$. A l'autre extrémité sont fixées en série 2 cartouches contenant chacune $200 \mathrm{mg}$ de Porapak Q. L'ensemble est alors placé dans une chambre climatisée et on y fait circuler un courant d'air de $1,51 / \mathrm{mn}$ pendant $24 \mathrm{~h}$ pour bien couvrir la totalité de la période d'appel des femelles. La $1^{\text {re }}$ cartouche, située en amont, filtre et purifie le courant d'air; les 2 autres cartouches, en aval, absorbent les émissions phéromonales des femelles en expérience. Elles sont ensuite extraites séparément par 3 fois $1 \mathrm{ml}$ d'hexane et la solution obtenue est concentrée à $100 \mu \mathrm{l}$ sous argon. Généralement, seul l'extrait de la cartouche immédiatement en aval du cylindre contient les produits phéromonaux.

Les chromatographies en phase gazeuse analytiques ont été réalisées :

- sur colonnes remplies (verre ou métal) avec un appareil Perkin-Elmer 3920 muni d'un détecteur à ionisation de flamme ;

- sur colonne capillaire de verre avec un appareil Girdel 300, muni d'un injecteur évaporateur de Ross et d'un détecteur à ionisation de flamme.

Les chromatographies en phase gazeuse préparatives ont été réalisées avec un appareil Perkin-Elmer 3920, muni d'un diviseur en sortie de colonne. Les échantillons ont été collectés dans des tubes capillaires en $\mathrm{U}$ refroidis dans l'azote liquide.

Les analyses par chromatographie en phase gazeuse couplée à la spectrométrie de masse (GC/MS) ont été réalisées sur un appareil NERMAG R-10-10 piloté par ordinateur (on utilisera les abréviations suivantes : EI impact électronique ; $\mathrm{CI}$ ionisation chimique).

Les observations comportementales ont été faites dans un olfactomètre à tube de verre, décrit par SOWER et al. (1973) et modifié par l'un d'entre nous (LALANNE-CASSOU, 1977), selon un protocole déjà décrit (RENOU et al., 1980).

Les produits de référence utilisés dans cette étude ont été synthétisés au Laboratoire des Médiateurs chimiques selon des procédés originaux (LETTERE, thèse de doctorat d'Université en cours) sauf pour l'octadécanal qui a été préparé par oxydation de l'octadécanol commercial (ALDRICH) avec le pyridinium chlorochromate dans le dichlorométhane.

On utilisera par la suite les abréviations suivantes :

Acétoxy-1 tétradécène-11Z : Z-11 TDA;

Acétoxy-1 tétradécène-11E : E-11 TDA;

Tétradécène-11Z al : Z-11 TDal ;

Tétradécène-11E al : E-11 TDal;

Tétradécène-11Z ol-1: Z-11 TDol ;

Octadécanal : $\mathrm{C}_{18} \mathrm{AL}$.

Les données numériques concernant l'efficacité sur les mâles des différentes fractions de l'extrait naturel 
et des produits de synthèse ont été soumises au test de DUNCAN. Il en a été de même pour les résultats des piégeages dans la nature, lorsqu'il a été possible aux expérimentateurs de répéter les essais un nombre suffisant de fois pour les traiter statistiquement.

\section{RÉSULTATS}

\section{A. Composition de la sécrétion phéromonale produite par les femelles vierges de $C$. pronubana}

L'analyse directe (fig. 1) par microencapsulation du contenu volatil de glandes à phéromone prélevées sur des femelles en appel montre la présence de 5 constituants principaux dont 3 se trouvent au niveau des membranes intersegmentaires indifférenciées de femelles ou de mâles analysées dans les mêmes conditions. Les 2 autres composés (pics 2 et 4 ) ont respectivement les temps de rétention du Z-11 TDA et du Z-11 TDol. Ils ne sont abondants que pendant la période d'appel des femelles, comme on peut le constater en prélevant des glandes sur des femelles du même âge tout au long du nyctémère et en les analysant dans les mêmes conditions.

L'analyse par chromatographie en phase gazeuse sur colonne capillaire de verre (fig. 2) d'un extrait hexanique obtenu à partir de 500 glandes, confirme l'existence de 2 produits (pics 4 et 5) aux temps de rétention du Z-11 TDA (65 p. 100) et du Z-11 TDol

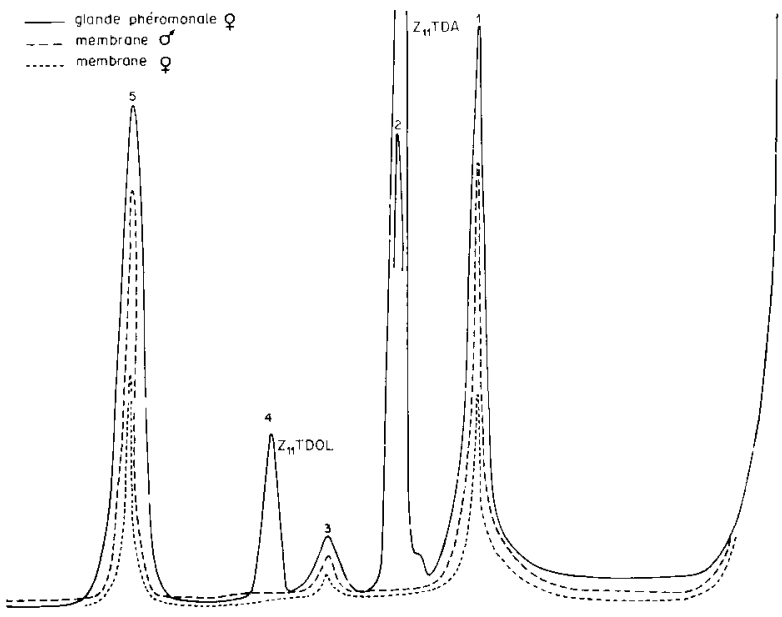

Figure 1

Analyse directe par microencapsulation (colonne inox $1 / 8$ " $5 m$ à 3 p. 100 FFAP sur Gas Chrom $6,180 \%$.

- - du contenu volatil de 8 glandes à phéromone de femelle vierge de C. pronubana âgée de $2 j$ pendant la période d'appel.

--- de 8 membranes intersegmentaires prélevées entre le $8^{e}$ et le $g^{e}$ segment abdominal sur des mâles naifs âgés de $2 j$ à une période correspondant à celle d'appel des femelles.

$\ldots .$. de 8 membranes intersegmentaires prélevées entre le bi et le $7^{e}$ segment abdominal sur des femelles vierges en appel âgées de $2 \mathrm{j}$.

Direct analysis by microencapsulation (column inox 1/8" $5 \mathrm{~m}$ impregnated with $3 \%$ FFAP on Gas Chrom G, $180^{\circ}$.

- - of the composition of volatiles from the sex pheromone glands of 8 2-days old virgin $\mathrm{C}$. pronubana females during the calling period.

--- of 8 intersegmentary membranes removed between the 8 th and 9 th abdominal segments of inexperienced males during a period corresponding to the « calling period 》 of virgin females.

of the intersegmentary membranes removed between the 6th and 7 th abdominal segments of virgin females (2 days old) during the calling period.

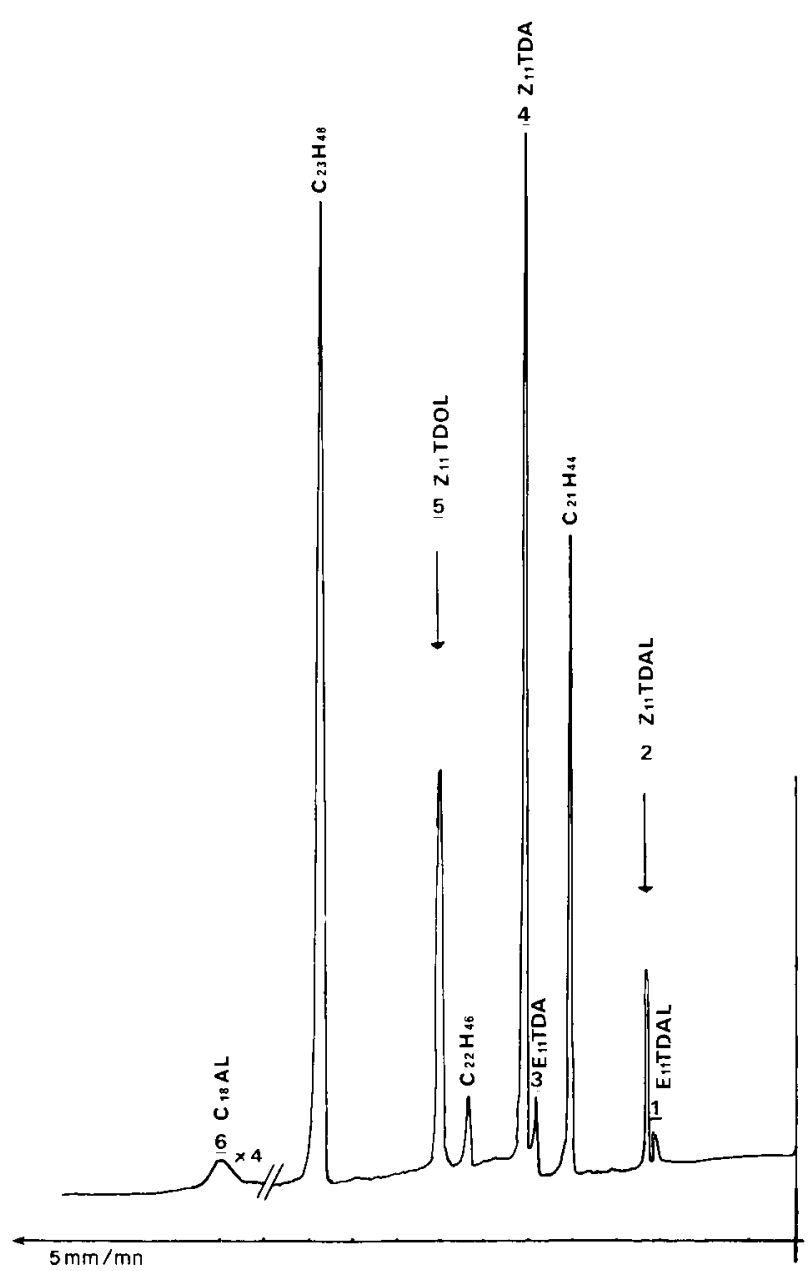

Figure 2

Analyses par chromatographie en phase gazeuse sur colonne capillaire de verre d'un extrait hexanique de 500 glandes à phéromone prélevées sur des femelles vierges de $\mathrm{C}$. pronubana en appel (colonne $50 \mathrm{~m}, 0,5 \mathrm{~mm}$ de diamètre intérieur, c. wax $20 \mathrm{M}, 175^{\circ} \mathrm{C}$ ). GC analysis on glass capillary column of an hexanic extract from 500 sex-pheromone glands of calling virgin females of $\mathrm{C}$. pronubana (column $50 \mathrm{~m}, 0.5 \mathrm{~mm} \mathrm{id}, \mathrm{c}$. $w a x 20 \mathrm{M}, 175^{\circ} \mathrm{C}$ ).

(20 p. 100) et de 4 nouveaux produits aux temps de rétention du E-11 TDal (pic $1 ; 2$ p. 100), du Z-11 TDal (pic 2; 10 p. 100), du E-11 TDA (pic 3; 3 p. 100) et du $C_{18} A L$ (pic $6<1$ p. 100) sans compter les pics attribuables aux hydrocarbures saturés de C21 à C-23 d'origine cuticulaire.

L'analyse par chromatographie en phase gazeuse couplée à la spectrométrie de masse du même extrait confirme les attributions proposées par les fragmentations caractéristiques suivantes:

a) Pour les tétradécénals : (pics 1 et 2); (E.I.), $\mathrm{m} / \mathrm{e}=192(\mathrm{M}-18) ;\left(\mathrm{C} . \mathrm{I} . / \mathrm{NH}_{3}\right), \mathrm{m} / \mathrm{e}=228(\mathrm{M}+18)$, $211(\mathrm{M}+1) ;\left(\mathrm{C} . \mathrm{I} . / \mathrm{CH}_{4}\right), \mathrm{m} / \mathrm{e}=251(\mathrm{M}+41), 239$ $(M+29), 211(M+1), 209(M-1), 193[(M+1)-18]$ et $191[(\mathrm{M}-1)-18]$.

b) Pour les acétates de tétradécényl : (pics 3 et 4 ) : (E.I.), $\mathrm{m} / \mathrm{e}=194(\mathrm{M}-60) ;\left(\right.$ C.I. $\left./ \mathrm{NH}_{3}\right) \mathrm{m} / \mathrm{e}=272$ $(\mathrm{M}+18), 255(\mathrm{M}+1) ;\left(\mathrm{C} . \mathrm{I} . / \mathrm{CH}_{4}\right) \mathrm{m} / \mathrm{e}=295$ $(M+41), 283(M+29), 255(M+1), 253(M-1)$, $195[(\mathrm{M}+1)-60]$ et $193[(\mathrm{M}-1)-60]$.

c) Pour le tétradécénol (pic 5) : (E.I.) $\mathrm{m} / \mathrm{e}=194$ $(\mathrm{M}-18) ;\left(\right.$ C.I. $\left./ \mathrm{NH}_{3}\right) \mathrm{m} / \mathrm{e}=230(\mathrm{M}+18)$, 
$213(\mathrm{M}+1) ;\left(\mathrm{C} . \mathrm{I} . / \mathrm{CH}_{4}\right), \mathrm{m} / \mathrm{e}=253(\mathrm{M}+41)$ $241(\mathrm{M}+29), 213(\mathrm{M}+1), 211(\mathrm{M}-1), 195[(\mathrm{M}+1)$ $-18]$ et 193 [ $(M-1)-18]$.

d) Pour l'octadécanal : (pic 6) : $\left(\right.$ C.I. $\left./ \mathrm{CH}_{4}\right) \mathrm{m} / \mathrm{e}=$ $309(\mathrm{M}+41), 297(\mathrm{M}+29), 269(\mathrm{M}+1), 267$ $(\mathrm{M}-1), 251[(\mathrm{M}+1)-18]$ et $249[(\mathrm{M}-1)-18]$.

On confirme par la même occasion les masses moléculaires des hydrocarbures saturés d'après les ions $(\mathrm{M}-1)$ correspondants en C.I./ $\mathrm{CH}_{4}$.

On confirme par la même occasion les masses moléculaires des hydrocarbures saturés d'après les ions $\left(\mathrm{M}^{+}-1\right)$ correspondants en (C.I./ $\left.\mathrm{CH}_{4}\right)$.

Afin de préciser la position et la géométrie de la double liaison présente dans les composés monoéthyléniques précédemment identifiés, on les a séparés en 3 fractions $\left(F_{1}, F_{2}, F_{3}\right)$ par chromatographie en phase gazeuse micropréparative (colonne inox $2 \mathrm{~m}, 1 / 8^{\prime \prime}$ à 5 p. 100 SE-30 sur V-30) et on a de nouveau analysé chaque fraction par chromatographie en phase gazeuse sur colonne capillaire de verre (colonne WCOT $50 \mathrm{~m}$, diamètre intérieur $0,5 \mathrm{~mm}$; Carbowax $20 \mathrm{M} ; 175{ }^{\circ} \mathrm{C}$ ). La fraction $\mathrm{F}_{1}$ présente 2 pics aux temps de rétention des Z-11 et E-11 TDal authentiques. La microozonolyse de cette fraction (BEROZA \& BIERL, 1967) donne uniquement de l'undécanedial (confirmé par GC/MS), ce qui permet de conclure que la double liaison initialement présente dans les 2 aldéhydes monoéthyléniques était bien en position 11 .

La fraction $F_{2}$ présente également 2 pics aux temps de rétention des Z-11 et E-11 TDA authentiques. Sa micro-époxydation (acide m-chloro perbenzöque/chlorure de méthylène) conduit à 2 époxydes dont l'analyse par GC/MS (colonne inox $1 / 8^{\prime \prime}$ à 3 p. 100 FFAP sur Gas Chrom G/HP à $220^{\circ} \mathrm{C}$ ) montre qu'ils proviennent de précurseurs monoéthyléniques possédant une double liaison en position 11 (présence de l'ion $241(\mathrm{M}-29)$ en E.I. et des ions $271(\mathrm{M}+1)$ et $288(\mathrm{M}+18)$ en C.I. $/ \mathrm{NH}_{3}$.

De plus l'époxyde majoritaire a le même temps de rétention que l'acétoxy- 1 cis époxy-11, 12 tétradécane authentique et le minoritaire celui de l'acétoxy-1 trans époxy-11, 12 tétradécane authentique.

La fraction $F_{3}$ ne présente qu'un seul pic au temps de rétention du Z-11 TDol et ne donne, après époxydation, qu'un seul époxyde provenant d'un alcool monoéthylénique possédant une double liaison en position 11 (présence de l'ion $199(\mathrm{M}-29)$ en E.I. et des ions $229(\mathrm{M}+1)$ et $246(\mathrm{M}+18)$ en C.I. $\left./ \mathrm{NH}_{3}\right)$. Cet époxyde ayant le même temps de rétention que le cis époxy-11,12 tétradécanol-1, on peut en conclure que le Z-11 TDol est bien l'alcool monoéthylénique en $C_{14}$ présent dans la sécrétion. Compte tenu de ce qui précède, la composition de l'extrait obtenu à partir des glandes à phéromone des femelles vierges de C. pronubana est la suivante : E-11 TDal (2 p. 100); Z-11 TDal (10 p. 100); E-11 TDA (3 p. 100) ; Z-11 TDA (65 p. 100) ; Z-11 TDol (20 p. 100) et C18 Al $(<1$ p. 100).

\section{B. Analyse des effluves odorants produits par les femelles vierges de $C$. pronubana en appel}

Afin de savoir si tous les composés précédemment identifiés étaient réellement émis par la femelle vierge dans les mêmes proportions, nous avons analysé des extraits obtenus par lavage à l'hexane de la glande à phéromone des femelles en appel (fig. 3) ou après adsorption sur Porapak $Q$ des effluves odorants produits par ces femelles (fig. 4). Dans les 2 cas, on retrouve les mêmes produits, mais on constate cependant que la proportion de Z-11 TDol (8 et 4 p. 100) est inférieure à ce qu'elle est dans l'extrait des glandes après macération, ce qui voudrait dire que ce produit pourrait servir de précurseur aux aldéhydes et acétates correspondants (WEATHERSTON \& MAC LEAN, 1974).

\section{Réactions comportementales des mâles de $C$. pro- nubana en olfactomètre}

Les réactions comportementales des mâles en olfactomètre ont été divisées en 2 séquences $R_{1}$ et $R_{2}$ ayant une signification sexuelle. $R_{1}$ correspond à un début d'orientation vers la source de phéromone, antennes relevées, pattes dressées et bourdonnement sur place. $\mathrm{R}_{2}$ correspond à un vol bourdonnant avec dévagination de la garniture génitale et tentatives d'accouplement avec l'applicateur (LALANNE-CASSOU, 1977).

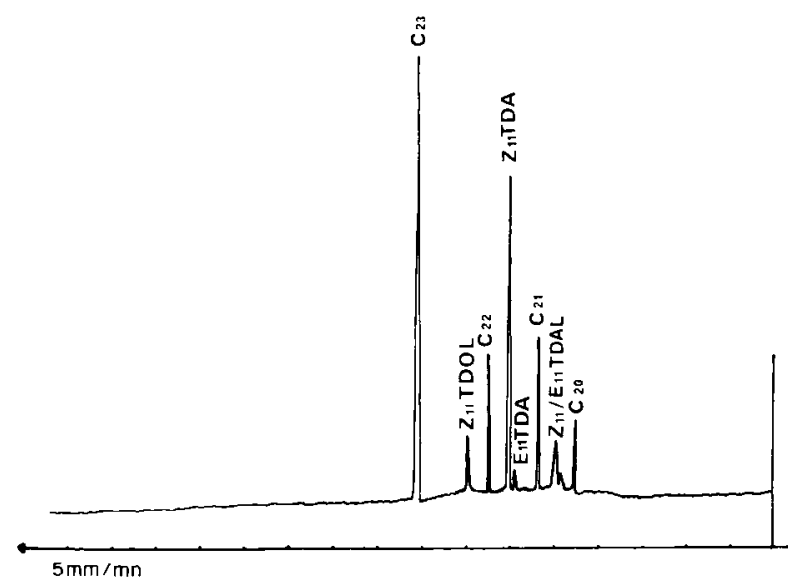

Figure 3

Chromatogramme obtenu après lavage à l'hexane de 5 glandes à phéromone de femelles vierges de C. pronubana en appel (colonne capillaire de verre $62 \mathrm{~m}, 0,5 \mathrm{~mm}$ de diamètre intérieur; c. wax $20 \mathrm{M} ; 195^{\circ} \mathrm{C}$ ).

$G C$ trace obtained after a wash with hexane from 5 sex pheromone glands of calling virgin females of $\mathrm{C}$. pronubana (glass capillary column $62 \mathrm{~m} ; 0.5 \mathrm{~mm}$ id ; c. $\operatorname{wax} 20 \mathrm{M} ; 195{ }^{\circ} \mathrm{C}$ ).

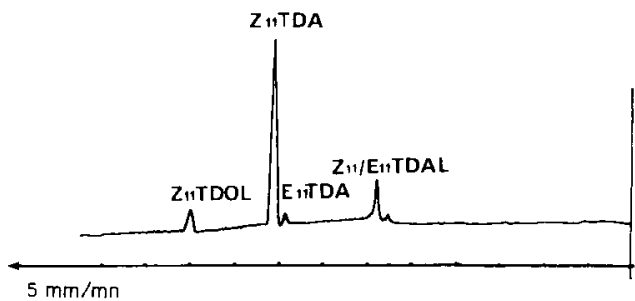

Figure 4

Chromatogramme obtenu après extraction à l'hexane des volatils adsorbés sur Porapak $Q$ pendant la période d'appel de 300 femelles vierges de C. pronubana (colonne capillaire de verre, $50 \mathrm{~m}, 0,5 \mathrm{~mm}$ de diamètre intérieur, c. wax $20 \mathrm{M} ; 195{ }^{\circ} \mathrm{C}$ ).

$G C$ trace obtained after extraction with hexane of volatiles adsorbed on Porapak $Q$ during the calling period of $300 \mathrm{C}$. pronubana virgin females (glass capillary column, $50 \mathrm{~m} ; 0.5 \mathrm{~mm}$ id; c. wax $20 \mathrm{M}$; $\left.195^{\circ} \mathrm{C}\right)$. 
On a d'abord éprouvé à la dose de $10^{-2}$ F.E. (F.E. = femelle équivalent ou quantité de phéromone d'un extrait correspondant à celle produite par 1 femelle vierge) l'extrait hexanique total des glandes à phéromone des femelles, puis, à la même concentration, les fractions $F_{1}, F_{2}, F_{3}$ précédentes correspondant à la chromatographie micropréparative du même extrait.

Les résultats obtenus sont rassemblés dans le tableau 1 . On constate que la fraction $F_{2}$, correspondant aux 2 acétates Z-11/E-11 TDA est à elle seule capable d'induire une réponse sexuelle de type $R_{2}$ chez 90 p. 100 des mâles en expérience. Par contre, la fraction $F_{1}$ (aldéhydes) est inactive et la fraction $F_{3}$ (alcool) moyennement ( 40 p. 100 de $R_{2}$ seulement). Le mélange $F_{2}+F_{3}$ (acétates + alcool) n'augmente pas globalement les réponses de type $R_{2}$ mais on observe cependant plus de tentatives d'accouplement avec l'applicateur ce qui voudrait dire que le Z-11 TDol pourrait intervenir sur le comportement à courte distance du mâle pendant la parade nuptiale.

Le mélange des 3 fractions $F_{1}+F_{2}+F_{3}$ reproduit tout à fait l'effet observé avec l'extrait total sans améliorer sensiblement le score obtenu avec le simple mélange $F_{2}+F_{3}$. Dans nos conditions d'expérimentation, il n'a pas été possible, en effet de mettre en évidence le rôle des aldéhydes (y compris l'octadécanal) sur le comportement sexuel des mâles. Des essais identiques conduits en chambre de vol pourraient peut-être apporter une réponse à cette question.

Les produits de synthèse, purs ou en mélange, ont été éprouvés dans les mêmes conditions à la dose de $100 \mathrm{pg}$. Les résultats obtenus sont rassemblés dans le tableau 2.

Les réponses de type $R_{1}$ au Z-11 TDA pur ( $>99$ p. 100) ou au mélange $Z-11 / E-11$ TDA $(97 / 3$ p. 100$)$ ne sont pas significativement différentes au seuil de 5 p. 100 ; par contre elles le sont pour le mélange $93 / 7$ p. 100 .

Les réponses de type $R_{2}$ au Z-11 TDA pur et aux 2 mélanges Z-11/E-11 TDA $(97 / 3$ et $93 / 7)$ sont significativement différentes entre elles.

Les réponses de type $R_{1}$ engendrées par les mélanges Z-11 TDA/Z-11 TDol (90/10 et 70/30) sont significativement différentes entre elles mais ne diffèrent pas des réponses au Z-11 TDA pur.

Les réponses de type $R_{2}$ engendrées par le Z-11 TDA pur et le mélange Z-11 TDA/Z-11 TDol $(90 / 10$ p. 100) sont significativement différentes entre elles.

Par contre, celles engendrées par le Z-11 TDA pur et le mélange $Z-11$ TDA/Z-11 TDol (70/30 p. 100) ne sont pas significativement différentes.

Ces résultats confirment l'importance du E-11 TDA (à des doses comprises entre 3 et 5 p. 100 par rapport au Z-11 TDA) dans l'orientation du mâle. Le Z-11 TDol (à des doses inférieures à 10 p. 100 par rapport au Z-11 TDA) n'agit pas de façon significative sur l'orientation du mâle, mais augmente son excitation sexuelle au contact de la source productrice de phéromone.

Le mélange ternaire Z-11 TDA/E-11 TDA (97/3) 90 p. 100 ; Z-11 TDol, 10 p. 100 reproduit pratiquement l'activité de l'extrait total des glandes à phéromone des femelles vierges éprouvé à une dose équiva-

\section{TABLEAU 1}

Réponses comportementales des mâles « naïfs " de C. pronubana $\grave{a}$ l'extrait total de glandes à phéromone de femelles et de différentes fractions obtenues par chromatographie préparative de cet extrait.

Behavioural responses of inexperienced $\mathrm{C}$. pronubana males to the total extract of sex pheromone glands from virgin females and to different fractions obtained after preparative gas chromatography.

\begin{tabular}{|c|c|c|c|c|}
\hline $\begin{array}{l}\text { Fractions testées } \\
\text { correspondant à la } \\
\text { dose de } 10^{-2} \mathrm{FE}\end{array}$ & $\begin{array}{l}\text { Nombre de } \\
\text { or « naïfs» } \\
\text { testés }\end{array}$ & $\begin{array}{l}\text { Nombre de } \\
\text { répétitions }\end{array}$ & $\begin{array}{l}\% 0 \\
R_{1}\end{array}$ & $\begin{array}{l}0 \% \\
\mathrm{R}_{2}\end{array}$ \\
\hline $\begin{array}{l}\text { Extrait hexanique total } \\
\text { de } \varphi \text { vierges }\end{array}$ & 50 & 3 & 100 & 92 \\
\hline Fraction $F_{1}$ (aldéhydes) & 25 & 2 & 0 & 0 \\
\hline Fraction $\mathrm{F}_{2}$ (acétates) & 25 & 2 & 90 & 90 \\
\hline Fraction $\mathrm{F}_{3}$ (alcool) & 20 & 2 & 60 & 40 \\
\hline$F_{1}+F_{2}$ & 20 & 2 & 70 & 70 \\
\hline $\mathrm{F}_{2}+\mathrm{F}_{3}$ & 10 & 3 & 100 & 90 \\
\hline$F_{1}+F_{2}+F_{3}$ & 10 & 3 & 100 & 91 \\
\hline
\end{tabular}

\section{TABLEAU 2}

Réponses en olfactomètre des mâles de C. pronubana à différents produits de synthèse, seuls ou en mélange, éprouvés à la dose de $100 \mathrm{pg}$.

Olfactometer responses of $\mathrm{C}$. pronubana males to various synthetic compounds alone or in mixture; test dose : $100 \mathrm{pg}$.

\begin{tabular}{lcrr}
\hline \hline Produits de synthèse & $\begin{array}{c}\text { Nombre de } \\
\text { o éprouvés }\end{array}$ & $\begin{array}{r}\% \\
\mathrm{R}_{1}\end{array}$ & $\begin{array}{r}\% \\
\mathrm{R}_{2}\end{array}$ \\
\hline Z-11 TDA & 30 & 79 & 15 \\
E-11 TDA & 30 & 0 & 0 \\
Z-11 TDol & 10 & 58 & 10 \\
Z-11 TDal & 10 & 30 & 20 \\
E-11 TDal & 10 & 0 & 0 \\
Z-11/E-11 TDA 97/3 & 130 & 89 & 85 \\
Z-11/E-11 TDA 93/7 & 20 & 60 & 40 \\
Z-11 TDA/Z-11 TDol 90/10 & 30 & 85 & 60 \\
Z-11 TDA/Z-11 TDol 70/30 & 30 & 60 & 45 \\
Z-11 TDA/Z-11 TDal 90/10 & 30 & 70 & 20 \\
(Z-11/E-11 TDA 97/3) + Z-11 & & & \\
TDol 90/10 & 30 & 95 & 90 \\
Z-11 TDal + (Z-11/E-11 TDA & & & \\
97/3) + Z-11 TDol 5/90/5 & 30 & 80 & 80 \\
Z-11 TDal + (Z-11/E-11 TDA & & & \\
97/3) + Z-11 TDol 10/80/10 & 30 & 80 & 75 \\
\hline \hline
\end{tabular}

lente $\left(10^{-2}\right.$ F.E.). Là encore, le rôle des Z-11/E-11 TDal n'a pu être mis en évidence, lorsqu'ils sont éprouvés à des doses voisines de celles trouvées dans les extraits.

\section{Piégeage des mâles de $C$. pronubana par des phé- romones sexuelles de synthèse}

Afin d'éprouver dans la nature l'efficacité des produits identifiés, on a mis en place, de 1978 à 1980, plusieurs réseaux de piégeage dans différentes localités du Sud-Est de la France spécialisées en cultures florales. Dans tous les cas, les produits de synthèse, à la dose de $1000 \mu \mathrm{g}$, sont adsorbés sur des capsules en caoutchouc (type bouchon pour tube à allergie, Ets Leune, Orsay) et les pièges sont du modèle I.N.R.A. (Ets Carol, Talence) utilisés avec leurs volets latéraux ouverts. Ils sont disposés à $20 \mathrm{~cm}$ environ au-dessus de la culture et changés de place tous les $8 \mathrm{j}$ par permutation circulaire. 
TABLEAU 3

Captures des mâles de C. pronubana réalisées en 1978 dans des pièges englués appâtés soit avec 5 femelles vierges en cagettes, renouvelées une fois par semaine, soit avec des formules suivantes:

$C A_{1}: Z-11 / E-11$ TDA $97 / 31000 \mu \mathrm{g}$

$C A_{2}: Z-11 / E-11$ TDA (97/3) $900 \mu \mathrm{g}+Z-11$ TDol $(100 \mu \mathrm{g})$

$C A_{3}: Z-11 / E-11$ TDA (97/3) $850 \mu g+Z-11$ TDol $(100 \mu g)+Z-11$ TDal $(50 \mu g)$.

Catches of male $C$. pronubana in sticky straps baited with 5 virgin females or with the following formulations :

$C A_{1}: Z-11 / E-11$ TDA $97 / 3 \quad 1000 \mu \mathrm{g}$

$C A_{2}: Z-11 / E-11 T D A(97 / 3) \quad 900 \mu g+Z-11 T D o l(100 \mu g)$

$C A_{3}: Z-11 / E-11 T D A(97 / 3) \quad 850 \mu g+Z-11 T D o l(100 \mu g)+Z-11 T D a l(50 \mu g)$.

\begin{tabular}{|c|c|c|c|c|c|c|c|c|}
\hline \multirow[b]{2}{*}{ dates des relevés } & \multicolumn{4}{|c|}{$\begin{array}{c}\text { Chambre d'Agriculture } \\
\text { d'Antibes }\end{array}$} & \multicolumn{3}{|c|}{ Hyères (Var) } & \multirow[b]{2}{*}{$\mathrm{CA}_{3}$} \\
\hline & $Q$ vierges & $\mathrm{CA}_{1}$ & $\mathrm{CA}_{2}$ & $\mathrm{CA}_{3}$ & $\$$ vierges & $\mathrm{CA}_{1}$ & $\mathrm{CA}_{2}$ & \\
\hline $13 / 7 / 1979$ & 19 & 23 & 59 & - & 5 & 1 & 40 & \\
\hline $25 / 7$ & 8 & 4 & 33 & - & 8 & 0 & 30 & \\
\hline $1 / 8$ & 25 & 4 & 31 & - & 6 & 0 & 59 & \\
\hline $8 / 8$ & 22 & 88 & 41 & - & 18 & 0 & 31 & \\
\hline $16 / 8$ & 7 & 73 & 40 & - & 10 & 4 & 7 & \\
\hline $23 / 8$ & 1 & 10 & 4 & - & 0 & 0 & 0 & \\
\hline $29 / 8$ & $i$ & 27 & 20 & - & 1 & 0 & 10 & \\
\hline $5 / 9$ & 20 & 48 & 44 & 51 & 1 & 6 & 13 & 13 \\
\hline $12 / 9$ & 4 & 117 & 63 & 160 & 0 & 1 & 61 & 29 \\
\hline $19 / 9$ & 12 & 48 & 39 & 72 & 14 & 0 & 8 & 21 \\
\hline Total & 119 & 442 & 374 & 283 & 63 & 12 & 259 & 63 \\
\hline
\end{tabular}

Les papillons capturés sont également dénombrés toutes les semaines et identifiés par leur armature génitale. Les capsules attractives sont remplacées toutes les 5 semaines en même temps que le fond englué du piège.

Les essais préliminaires de 1978, conduits du 13 juillet au 19 septembre (et du 5 au 19 septembre pour la formulation $\mathrm{CA}_{3}$ ) dans les roseraies du service de vulgarisation de la Chambre d'Agriculture d'Antibes (Alpes-Maritimes) et dans les cultures d'œillets à Hyères (Var), ont montré (tabl. 3) que le mélange Z11/E-11 TDA 97/3 était attractif (même supérieur aux femelles vierges à Antibes) et que l'addition du Z-11 TDol n'avait pas toujours le même effet selon la localité considérée.

En 1979, des essais ont été conduits seulement à la Chambre d'Agriculture d'Antibes mais la période de piégeage a été plus longue (du 5 juin au 20 août) et les formulations utilisées plus nombreuses (tabl. 4).

Nous n'avons pas repris cette année-là les formulations contenant $\mathrm{du} \mathrm{Z}$-11 TDal car nous voulions d'abord confirmer le rôle des E-11 TDA et Z-11 TDol en mélange avec le Z-11 TDA et étudier l'éventuel effet inhibiteur du Z-9 TDA, constituant minoritaire de la sécrétion phéromonale d'Epichoristodes acerbella (Walk.) afin d'éviter les captures indésirables des mâles de $C$. pronubana dans les pièges à $E$. acerbella (captures particulièrement gênantes à cause de la ressemblance des 2 insectes dans les pièges englués).

On constate là encore que le mélange $\mathrm{Z}-11 / \mathrm{E}-11$ TDA 97/3 attire fortement les mâles et que l'adjonction de quantités croissantes de E-11 TDA (jusqu'à 20 p. 100) diminue significativement les captures.

Lorsqu'on ajoute le Z-11 TDol au mélange précédent, $\left(\mathrm{CP}_{4}\right)$ c'est-à-dire lorsqu'on se rapproche de la composition présumée de la phéromone de la femelle, on n'augmente pas de façon significative les captures. (Moyenne : 19,7 pour $\mathrm{CP}_{1}, 20$ pour $\mathrm{CP}_{4}$ ).

Par ailleurs, l'addition de Z-9 TDA au Z-11/E-11 TDA 97/3 diminue significativement l'attraction des

\section{TABLEAU 4}

Captures des mâles de C. pronubana (moyenne par semaine) dans différents pièges englués chargés des formulations suivantes: $C P_{1}:$ Z-11/E-11 TDA 97/3 $1000 \mu \mathrm{g}$ $C P_{2}:$ Z-11/E-11 TDA 90/10 $1000 \mu \mathrm{g}$ $\mathrm{CP}_{3}:$ Z-11/E-11 TDA 80/20 $1000 \mu \mathrm{g}$ $C P_{4}:(Z-11 / E-11 T D A$ 97/3) $900 \mu g+Z-11$ TDol $100 \mu g$ $C P_{5}:(Z-11 / E-11 T D A 97 / 3) \quad 970 \mu g+Z-9$ TDA $30 \mu g$ $C P_{6}:(Z-11 / E-11 T D A$ 97/3) $\quad 800 \mu \mathrm{g}+$ Z-9 TDA $200 \mu \mathrm{g}$ Résultats de 1979 ; chambre d'Agriculture d'Antibes.

Catches of C. pronubana males in sticky traps (average per week) baited with the following formulations :

$C P_{1}$ : Z-11/E-11 TDA 97/3 $1000 \mu \mathrm{g}$

$C P_{2}:$ Z-11/E-11 TDA $90 / 101000 \mu \mathrm{g}$

$C P_{3}:$ Z-11/E-11 TDA 80/20 I $000 \mu \mathrm{g}$

$C P_{4}:(Z-11 / E-11$ TDA 97/3) $900 \mu g+Z-11$ TDol $100 \mu g$ $C P_{5}:(Z-11 / E-11$ TDA 97/3) $970 \mu \mathrm{g}+Z-9$ TDA $30 \mu \mathrm{g}$ $C P_{6}:(Z-11 / E-11$ TDA 97/3) $800 \mu \mathrm{g}+Z-9$ TDA $200 \mu \mathrm{g}$ Results of 1979 ; Antibes, «Chambre d'Agriculture ».

\begin{tabular}{|c|c|c|c|c|c|c|}
\hline Formulations utilisées & $\mathrm{CP}_{1}$ & $\mathrm{CP}_{2}$ & $\mathrm{CP}_{3}$ & $\mathrm{CP}_{4}$ & $\mathrm{CP}_{5}$ & $\mathrm{CP}_{6}$ \\
\hline $\begin{array}{l}\text { Durée du piégeage } \\
\text { (en semaines) }\end{array}$ & 12 & 12 & 12 & 12 & 12 & 12 \\
\hline $\begin{array}{l}\text { Nombre de } \sigma^{\circ} \text { capturés } \\
\text { en moyenne }\end{array}$ & & & & & & \\
\hline par semaine & 19,7 & 13,8 & 11,3 & 20 & 7,2 & 0,3 \\
\hline
\end{tabular}

mâles jusqu'à l'inhiber presque complètement à forte dose.

En 1980, les essais repris par le Service de la Protection des Végétaux d'Hyères (Var) dans des cultures mixtes d'œillets et de rosiers, avaient pour but de compléter les résultats précédents et de mettre en évidence le rôle éventuel des différents aldéhydes identifiés dans la sécrétion (tabl. 5). L'addition de Z-11 TDal (formulation $\mathrm{C}_{2}$ ) ou de E-11 TDal (formulation $\mathrm{C}_{3}$ ) ou de leur mélange (formulation $\mathrm{C}_{4}$ ) au mélange Z-11/E-11 TDA $97 / 3$ pris comme témoin, augmente significativement les captures sans qu'il soit possible ni de déterminer l'isomère géométrique le plus efficace ni de mettre en évidence une synergie de l'un sur l'autre. 


\section{TABLEAU 5}

Captures des mâles de C. pronubana dans des pièges englués chargés des formulations suivantes:

$C_{1}: Z-11$ TDA/E-11 TDA 97/3 $900 \mu \mathrm{g}+Z-11$ TDol $100 \mu \mathrm{g}$ $C_{2}: Z-11$ TDA/E-11 TDA 97/3 $900 \mu \mathrm{g}+Z-11$ TDal $100 \mu \mathrm{g}$ $C_{3}$ : Z-11 TDA/E-11 TDA 97/3 $900 \mu \mathrm{g}+$ E-11 TDal $100 \mu \mathrm{g}$ $C_{4}: Z-11$ TDA/E-11 TDA 97/3 $900 \mu g+$ Z-11 TDal/E-11 TDal $50 / 50100 \mu \mathrm{g}$

$C_{5}:$ Z-11 TDA/E-11 TDA 97/3 $900 \mu \mathrm{g}+$ Z-11 TDal/E-11

TDal 50/50 $50 \mu \mathrm{g}+Z-11 \mathrm{TDol} 50 \mu \mathrm{g}$.

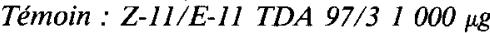

(Résultats de 1980, Protection des Végétaux, Hyères).

Catches of $\mathrm{C}$. pronubana males with the following formulations : $C_{1}$ : Z-11 TDA/E-1I TDA 97/3 $900 \mu \mathrm{g}+$ Z-11 TDol $100 \mu \mathrm{g}$ $C_{2}:$ Z-11 TDA/E-11 TDA 97/3 $900 \mu \mathrm{g}+$ Z-11 TDal $100 \mu \mathrm{g}$ $C_{3}: Z-11 T D A / E-11 T D A 97 / 3 \quad 900 \mu g+E-11 T D a l 100 \mu g$ $C_{4}: Z-11 T D A / E-11 T D A 97 / 3 \quad 900 \mu \mathrm{g}+Z-11$ TDal/E-11 TDal $50 / 50100 \mu \mathrm{g}$

$C_{5}:$ Z-11 TDA/E-11 TDA 97/3 $900 \mu g+Z-11$ TDal/E-11

TDal $50 / 5050 \mu \mathrm{g}+Z-11$ TDol $50 \mu \mathrm{g}$.

Blank : Z-11/E-11 TDA 97/3 $1000 \mu \mathrm{g}$

(Results of 1980. Plant Protection Agency Hyères).

\begin{tabular}{lrrrrrr}
\hline \multicolumn{1}{c}{ Formulations } & & & & & & \\
Dates des relevés & $C_{1}$ & $C_{2}$ & $C_{3}$ & $C_{4}$ & $C_{5}$ & Témoin \\
\hline $31 / 7 / 1980$ & & $\ldots$ & & & & \\
$4 / 8$ & 26 & 72 & 48 & 42 & 5 & 22 \\
$14 / 8$ & 50 & 33 & 46 & 12 & 6 & 15 \\
$22 / 8$ & 6 & 38 & 24 & 2 & 1 & 15 \\
$29 / 8$ & 5 & 21 & 11 & 16 & 4 & 8 \\
$5 / 9$ & 6 & 9 & 5 & 10 & 15 & 7 \\
$12 / 9$ & 0 & 5 & 7 & 4 & 8 & 6 \\
$19 / 9$ & 11 & 41 & 146 & 61 & 3 & 31 \\
$26 / 9$ & 51 & 49 & 63 & 47 & 4 & 32 \\
$4 / 10$ & 3 & 21 & 28 & 31 & 0 & 25 \\
$10 / 10$ & 6 & 2 & 12 & 62 & 5 & 8 \\
Nombre total des & 1 & 4 & 6 & 3 & 3 & 3 \\
mâles capturés & & & & & & \\
\hline \hline
\end{tabular}

Dans les 3 cas, l'augmentation des captures observées n'est pas significativement supérieure à celle qu'aurait donné l'adjonction d'une quantité équivalente de Z-11 TDol (formulation $\mathrm{C}_{1}$ ).

Par contre, le mélange des aldéhydes et des alcools additionné au mélange Z-11/E-11 TDA diminue sensiblement les captures (formulation $\mathrm{C}_{5}$ ), sans qu'une explication puisse être donnée à cette observation.

Ces différentes campagnes de piégeage confirment en partie les résultats des tests olfactométriques de laboratoire. Dans les 2 cas, on a pu montrer l'effet attractif du mélange Z-11/E-11 TDA (compris entre $97 / 3$ et $95 / 5$ ) et un faible effet synergiste du Z-11 TDol (entre 6 et 10 p. 100).

Par contre, l'effet synergiste des aldéhydes (Z-11 TDal ou E-11 TDal) n'a pas été confirmé au laboratoire dans les conditions d'expériences utilisées.

Pour des raisons pratiques (stabilité des produits), les capsules attractives proposées pour piéger les mâles de C. pronubana ont la composition suivante : mélange Z-11 TDA/E-11 TDA (97/3) 90 p. $100 ;$ Z-11 TDol, 10 p. 100, charge totale par capsule $1000 \mu \mathrm{g}$.

\section{E. Spécificité des attractifs de synthèse}

Toutes les formulations éprouvées se sont révélées assez spécifiques; cependant, en début de vol (juin- juillet), on peut capturer des mâles de la tordeuse verte du chêne (Tortrix viridana L.), surtout si des chênes de différentes espèces sont présents dans les sites de piégeage.

Enfin, la présence de vergers ou de vignobles peut favoriser également, en début de campagne, la capture d'une autre tordeuse Archipini, Argyrotaenia pulchellana (Haw), surtout dans le Sud-Est de la France où les périodes d'activité des 2 insectes coïncident. Dans nos conditions de piégeages, ces captures indésirables n'ont jamais dépassé 1 p. 100 de l'ensemble des captures.

\section{CONCLUSION}

Comme l'ensemble des autres Archipini étudiés, les femelles de $C$. pronubana utilisent le Z-11 TDA comme constituant principal de leur sécrétion phéromonale. La présence de quelques p. 100 de E-11 TDA rapprocherait $C$. pronubana des autres tordeuses Archipini telles que Argyrotaenia velutinana (Walk.) (ROELOFS et al., 1976b) et Archips mortuanus (Kearf.) (CARDE et al., 1977), pour lesquelles le E-11 TDA est aussi le composé minoritaire de leur sécrétion phéromonale et le Z-9 TDA un inhibiteur total ou partiel. Par contre, l'espèce se distinguerait de celles pour lesquelles la situation est inverse telles que Pandemis limitata (Rob.) (ROELOFS et al., 1976a) $P$. pyrusana (Kaw.) (RoElors et al., 1977) P. heparana (Den. \& Schiff.) (FREROT et al., 1982).

C'est la raison pour laquelle on ne capture jamais $E$. acerbella dans les pièges à $C$. pronubana, le E-11 TDA étant inhibiteur des captures de $E$. acerbella dès 0,1 p. 100 (LALANNE-CASSOU \& FREROT, 1980) L'inverse n'est cependant pas vrai car le Z-9 TDA, présent à 8 p. 100 dans la sécrétion de $E$. acerbella, n'est pas encore fortement inhibiteur, à cette dose, des captures de mâles de $C$. pronubana.

Tout se passerait donc comme si les Z-11 TDA/E-11 TDA (en mélange $97 / 3$ ou 95/5) étaient nécessaires à l'attraction à distance des mâles de $C$. pronubana par les femelles vierges en appel. C'est en suivant cette piste odorante que le mâle se rapprocherait de sa partenaire et en reconnaîtrait l'identité. Par contre le Z11 TDol n'interviendrait pas directement dans cette attraction mais pourrait favoriser le déroulement de la parade nuptiale, lorsque les 2 partenaires sont déjà l'un près de l'autre. Cette hypothèse devrait néanmoins être confirmée par des observations comportementales dans la nature (table de vol) ou au laboratoire (chambre de vol). Le Z-9 TDA n'est par contre pas suffisamment inhibiteur dans l'attraction des mâles de $C$. pronubana pour que ceux-ci ne soient pas attirés par des capsules destinées au piégeage des mâles de E. acerbella ou d'autres Archipini utilisant ce composé dans leur message phéromonal. L'exploration électrophysiologique, au niveau unitaire, des récepteurs olfactifs des antennes des mâles de $C$. pronubana (PRIESNER, 1979a, $b$ ) a permis cependant de découvrir des récepteurs spécifiques au Z-9 TDA, ce qui signifie que les antennes des mâles perçoivent non seulement les constituants élémentaires du bouquet 
phéromonal de leur propre femelle mais aussi ceux des femelles des autres espèces sympatriques qui ont coévolué avec eux. Cette observation rend compte, au niveau moléculaire, de la pression de sélection que peuvent exercer les espèces les unes sur les autres à l'intérieur d'une biocœnose.

\section{REMERCIEMENTS}

Nous remercions Mr J. M. Gailleron (Service de la Protection des végétaux d'Hyères) pour l'aide qu'il nous a apportée dans la réalisation des piégeages en 1980 et la Chambre d'Agriculture d'Antibes pour avoir mis ses roseraies à notre disposition.

Reçu le 7 juin 1983 Accepté le 12 juillet 1984

\section{RÉFÉRENCES BIBLIOGRAPHIQUES}

Beroza M., Bierl A. B., 1967. Rapid determination of olefin position in organic compounds in microgram range by ozonolysis and gas chromatography. Anal. Chem., 39, 1131-1135.

Bovey P., 1966. Super famille des Tortricidae. In Balachowsky A. S. : « Entomologie appliquée à l'agriculture Tome II (I re partie) », Masson et Cie éd., 456-893.

Carde R. T., Carde A. M., Hill A. S., Roelofs W. L., 1977. Sex pheromone specificity as a reproductive isolating mechanism among the sibling species Archips argyrospilus and $A$. mortuanus and other sympatric tortricine moths (Lepidoptera Tortricidae). J. Chem. Ecol., 3 (1), $71-74$

Delucchi V. I., Merle L., 1962. La tordeuse de l'œillet Cacaecimorpha pronubana (Hübner) (Lépidoptère, Tortricidae), ravageur peu connu des agrumes au Maroc. Al Awamia, 3, 79-86.

Descoins C., Gallois M., 1979. Analyse directe par chromatographie en phase gazeuse des constituants volatils présents dans les glandes à phéromone des femelles de lépidoptères. Ann. Zool. Ecol. Anim., 11 (4), 521-532.

Frerot B., Gallois M., Lettere M., Einhorn J., Michelot D., Descoins C., 1982. Sex pheromone of Pandemis heparana (Den. \& Schiff.) (Lepidoptera, Tortricidae). J. Chem. Ecol., 8 (3), 663-670.

Klun J. A., Plimmer J. R., Bierl-Leonhardt A. B., Sparks A. M., Chapman O. L., 1979. Trace chemicals : the essence of sexual communication systems in Heliothis species. Science, 204 (4399), 13281330.

Lalanne-Cassou B., 1977. Contribution à l'étude de la communication sexuelle par phéromone chez l'eudemis de la vigne: Lobesia botrana (Schiff.) (Lépidoptère, Tortricidae, Olethreutinae). Thèse Doc. $3^{\text {e }}$ cycle, Biologie animale, option Entomologie, Université Pierre et Marie Curie, Paris, 247 p.

Lalanne-Cassou B., Frerot B., 1980. Analyse de la sécrétion phéromonale de la tordeuse sud-africaine de l'oeillet: Epichoristodes acerbella (Walk.) (Lépidoptère, Tortricidae). C. R. Acad. Sci., Sér. $D, 291,845-848$.
Millière P., 1864. Iconographie et description de chenilles de lépidoptères inédits ( $T$. pronubana), Ann. Soc. Linn. Lyon, 382-385.

Pralavorio M., Millot P., 1978. Biologie et écologie de la tordeuse, Epichoristodes acerbella (Walk.) (Lepidoptera, Tortricidae). Ann. Zool. Ecol. Anim., 10 (4), 645-661.

Priesner E., 1979a. Specificity studies on pheromone receptors of noctuid and tortricid lepidoptera, 57-71. In Ritter F. J. : " Chemical ecology : Odour communication in animals », Elsevier, Amsterdam, $427 \mathrm{p}$

Priesner E., 1979b. Progress in the analysis of pheromone receptor systems. Ann. Zool. Ecol. Anim., 11 (4), 533-546.

Renou M., Descoins C., Lallemand J. Y., Priesner E., Lettere M., Gallois M., 1980. L'acétoxy-1 dodécène-3E, composant principal de la phéromone sexuelle de la teigne de la betterave : Scrobipalpa acellatella (Boyd.) (Lépidoptère, Gelechiidae). Z. Angew. Entomol., 90, 275-289.

Roelofs W. L., Carde A., Hill A., Carde R. T., 1976a. Sex pheromone of the threelined leafroller : Pandemis limitata. Environ. Entomol., 5 (4), 649-652.

Roelofs W., Hill A., Carde R. T., 1976b. Sex pheromone components of the red banded leafroller : Argyrotaenia velutinana (Lepidoptera, Tortricidae). J. Chem. Ecol., 1, 83-89.

Roelofs W. L., Lagier R. F., Hoyl S. C., 1977. Sex pheromone of the moth, Pandemis pyrusana. Environ. Entomol., 6 (3), 353-354.

Sower L., Vick K. W., Long J. S., 1973. Isolation and preliminary biological studies of the female produced sex pheromone of Sitotroga cerealella (Lepidoptera, Gelechiidae). Ann. Entomol. Soc. Am., 66, 184-187.

Targe A., Deportes L., 1961. La tordeuse de l'œillet, Phytoma, $128,13-17$ et $129,17-22$.

Wheatherston J. W., Mac Lean W., 1974. The occurrence of (E)11-tetradecene 1-ol a known sex attractant inhibitor, in the abdominal tips of virgin females eastern spruce budworm, Choristoneura fumiferana (Lepidoptera Tortricidae). Can Entomol., 106, 281-284. 